The following resources related to this article are available online at www.sciencemag.org (this information is current as of October 14, 2009 ):

Updated information and services, including high-resolution figures, can be found in the online version of this article at:

http://www.sciencemag.org/cgi/content/full/326/5949/74

Supporting Online Material can be found at:

http://www.sciencemag.org/cgi/content/full/326/5949/74/DC1

A list of selected additional articles on the Science Web sites related to this article can be found at:

http://www.sciencemag.org/cgi/content/full/326/5949/74\#related-content

This article cites $\mathbf{8 6}$ articles, 23 of which can be accessed for free:

http://www.sciencemag.org/cgi/content/full/326/5949/74\#otherarticles

This article has been cited by 1 articles hosted by HighWire Press; see:

http://www.sciencemag.org/cgi/content/full/326/5949/74\#otherarticles

This article appears in the following subject collections:

Anthropology

http://www.sciencemag.org/cgi/collection/anthro

Information about obtaining reprints of this article or about obtaining permission to reproduce this article in whole or in part can be found at:

http://www.sciencemag.org/about/permissions.dtl 


\title{
Reexamining Human Origins in Light of Ardipithecus ramidus
}

\author{
C. Owen Lovejoy
}

$\mathrm{C}$ impanzees, bonobos, and gorillas are our closest living relatives. The most popular reconstructions of human evolution during the past century rested on the presumption that the behaviors of the earliest hominids were related to (or even natural amplifications of) behaviors observed in these living great apes. One effect of chimpanzee-centric models of human evolution has been a tendency to view Australopithecus as transitional between an ape-like ancestor and early Homo.

Ardipithecus ramidus nullifies these presumptions, as it shows that the anatomy of living African apes is not primitive but instead has evolved specifically within extant ape lineages. The anatomy and behavior of early hominids are therefore unlikely to represent simple amplifications of those shared with modern apes. Instead, $A r$. ramidus preserves some of the ancestral characteristics of the last common

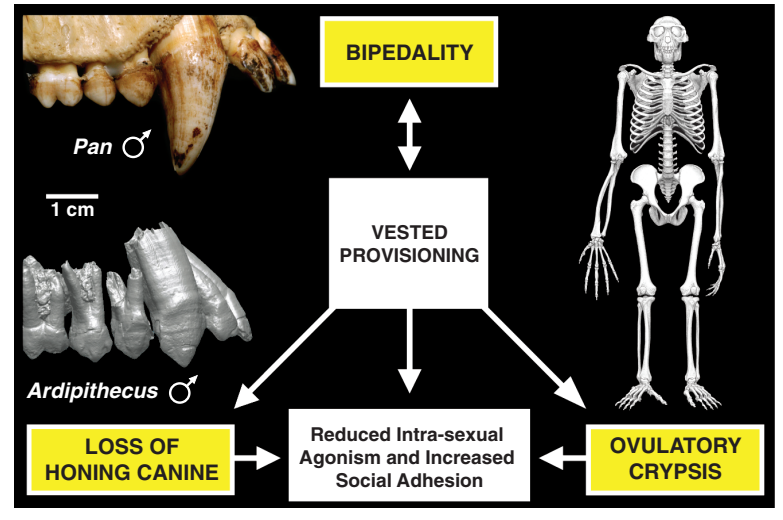

Breakthrough adaptations can transform life-history by deviating from typical reproductive strategy. Early hominids show feminized male canines [left] and primitive bipedality [right]. These suggest that females preferred nonaggressive males who gained reproductive success by obtaining copulation in exchange for valuable foods (vested provisioning). Success would depend on copulatory frequency with mates whose fertility remained cryptic (e.g., absence of cycling in mammary size). The result would be reduced agonism in unrelated females, and cooperative expansion of day ranges among equally cooperative males, eventually leading to exploitation of new habitats. ancestor with much greater fidelity than do living African apes. Two obvious exceptions are its ability to walk upright and the absence of the large projecting canine tooth in males, derived features that Ardipithecus shares with all later hominids.

Ar. ramidus illuminates our own origins because it clarifies our relationship to Australopithecus. For example, the enlarged rear teeth of Australopithecus have long been viewed as adaptations to a rough, abrasive diet. This has led to speculation that canine teeth might have become smaller simply to accommodate the emergence of these other enlarged teeth, or that the importance of canine teeth in displays of male-to-male aggression waned with the development of weapons. Ar. ramidus negates such hypotheses because it demonstrates that small canines occurred in hominids long before any of the dental modifications of Australopithecus or the use of stone tools. The loss of large canine teeth in males must have occurred within the context of a generalized, nonspecialized diet. Comparisons of the Ar. ramidus dentition with those of all other higher primates indicate that the species retained virtually no anatomical correlates of male-to-male conflict. Consistent with a diminished role of such agonism, the body size of Ar. ramidus males was only slightly larger than that of females.

The discovery of Ar. ramidus also requires rejection of theories that presume a chimpanzee- or gorilla-like ancestor to explain habitual upright walking. Ar. ramidus was fully capable of bipedality and had evolved a substantially modified pelvis and foot with which to walk upright. At the same time, it preserved the ability to maneuver in trees, because it maintained a grasping big toe and a powerful hip and thigh musculature. Because upright walking provided no energy advantage for Ar. ramidus (it lacked many of the adaptations evolved in later hominids such as Australopithecus), reproductive success must have been central to its evolution in early hominids.

Loss of the projecting canine raises other vexing questions because this tooth is so fundamental to reproductive success in higher primates. What could cause males to forfeit their ability to aggressively compete with other males? What changes paved the way for the later emergence of the energythirsty brain of Homo? Such questions can no longer be addressed by simply comparing humans to extant apes, because no ape exhibits an even remotely similar evolutionary trajectory to that revealed by Ardipithecus.

When the likely adaptations of early hominids are viewed generally rather than with specific reference to living chimpanzees, answers to such questions arise naturally. Many odd hominid characteristics become transformed from peculiar to commonplace. Combining our knowledge of mammalian reproductive physiology and the hominid fossil record suggests that a major shift in life-history strategy transformed the social structure of early hominids. That shift probably reduced male-to-male conflict and combined three previously unseen behaviors associated with their ability to exploit both trees and the land surface: (i) regular food-carrying, (ii) pair-bonding, and (iii) reproductive crypsis (in which females did not advertise ovulation, unlike the case in chimpanzees). Together, these behaviors would have substantially intensified male parental investment — a breakthrough adaptation with anatomical, behavioral, and physiological consequences for early hominids and for all of their descendants, including ourselves.

When citing, please refer to the full paper, available at DOI 10.1126/science.1175834. 


\section{Reexamining Human Origins in Light of Ardipithecus ramidus}

\section{Owen Lovejoy}

Referential models based on extant African apes have dominated reconstructions of early human evolution since Darwin's time. These models visualize fundamental human behaviors as intensifications of behaviors observed in living chimpanzees and/or gorillas (for instance, upright feeding, male dominance displays, tool use, culture, hunting, and warfare). Ardipithecus essentially falsifies such models, because extant apes are highly derived relative to our last common ancestors. Moreover, uniquely derived hominid characters, especially those of locomotion and canine reduction, appear to have emerged shortly after the hominid/chimpanzee divergence. Hence, Ardipithecus provides a new window through which to view our clade's earliest evolution and its ecological context. Early hominids and extant apes are remarkably divergent in many cardinal characters. We can no longer rely on homologies with African apes for accounts of our origins and must turn instead to general evolutionary theory. A proposed adaptive suite for the emergence of Ardipithecus from the last common ancestor that we shared with chimpanzees accounts for these principal ape/human differences, as well as the marked demographic success and cognitive efflorescence of later Plio-Pleistocene hominids.

A n essential goal of human evolutionary studies is to account for human uniqueness, most notably our bipedality, marked demographic success, unusual reproductive physiology, and unparalleled cerebral and technological abilities. During the past several decades, it has been routinely argued that these hominid characters have evolved by simple modifications of homologs shared with our nearest living relatives, the chimpanzee and bonobo. This method is termed referential modeling (1). A central tenet has been the presumption (sometimes clearly stated but more often simply sub rosa) that Gorilla and Pan are so unusual and so similar to each other that they cannot have evolved in parallel; therefore, the earliest hominids must have also resembled these African apes $(2,3)$. Without a true early hominid fossil record, the de facto null hypothesis has been that Australopithecus was largely a bipedal manifestation of an African ape (especially the chimpanzee). Such proxy-based scenarios have been elevated to common wisdom by genomic comparisons, progressively establishing the phylogenetic relationships of Gorilla, Pan, and Homo (4).

Early Australopithecus. Although Australopithecus was first encountered early in the last century (5), its biology was only slowly revealed. In the 1970s, abundant earlier Australopithecus fossils began to emerge in eastern Africa. These samples broadened our understanding of the genus and included partial skeletons (6) and even footprint trails [the latter extending our knowledge to 3.75 million years ago (Ma)] (7).

Department of Anthropology, School of Biomedical Sciences, Kent State University, Kent, OH 44242-0001, USA. E-mail: olovejoy@aol.com
To many, these fossils were consistent with chimpanzee-based referential scenarios. Bipedality had long been argued to have occurred when early hominids ventured onto the expanding savannas and grasslands of the Pliocene $(8,9)$. More recently, bipedality is seen to have emerged from African ape behaviors, including feeding postures $(10,11)$, gorilla dominance displays (12), and even vertical climbing (13). Many mechanical/behavioral models have been proposed to explain the evolution of hominid bipedality, but most have presumed it to have evolved from a chimpanzee-like ancestor $(4,14,15)$. A primary problem with these scenarios has been the remarkably advanced postcranium of early Australopithecus, which exhibits particularly advanced adaptations to upright walking (16-18).

Ardipithecus ramidus. Ardipithecus ramidus now reveals that the early hominid evolutionary trajectory differed profoundly from those of our ape relatives from our clade's very beginning. Ar. ramidus was already well-adapted to bipedality, even though it retained arboreal capabilities (19-25). Its postcranial anatomy reveals that locomotion in the chimpanzee/human last common ancestor (hereafter the CLCA) must have retained generalized above-branch quadrupedality, never relying sufficiently on suspension, vertical climbing, or knuckle walking to have elicited any musculoskeletal adaptations to these behaviors (26-28).

Moreover, Ardipithecus was neither a ripefruit specialist like Pan, nor a folivorous browser like Gorilla, but rather a more generalized omnivore $(19,25)$. It had already abandoned entirely the otherwise universal sectorial canine complex (SCC), in which the larger, projecting upper canine is constantly honed by occlusion against the lower third molar of anthropoid primates (25), demonstrating that the large, projecting, interlocking, and honing male canines of apes had been eliminated before the dawn of the Pliocene and before the emergence of the dentognathic peculiarities of Australopithecus. What's more, it appears to have been only slightly dimorphic in body size (25). Finally, the environmental context of Ardipithecus suggests that its primary habitat was not savanna or grassland, but instead woodlands (26-28).

In retrospect, clues to this vast divide between the evolutionary trajectories of African apes and hominids have always been present. Apes are largely inept at walking upright. They exhibit reproductive behavior and anatomy profoundly unlike those of humans. African ape males have retained (or evolved, see below) a massive SCC and exhibit little or no direct investment in their offspring (their reproductive strategies rely primarily on varying forms of male-to-male agonism). Although they excel at some cognitive tasks, they perform at levels qualitatively similar to those of some extraordinary birds $(29,30)$ and mammals (31). The great apes are an isolated, uniquely specialized relict species surviving today only by their occupation of forest refugia (32). Even their gut structure differs substantially from that of humans (33).

How and why did such profound differences between hominids and African apes evolve? Why did early hominids become the only primate to completely eliminate the SCC? Why did they become bipedal, a form of locomotion with virtually no measurable mechanical advantage (34)? Why did body-size dimorphism increase in their likely descendants? These are now among the ultimate questions of human evolution. We can, of course, only hypothesize their answers. Nevertheless, by illuminating the likely morphological structure and potential social behavior of the CLCA, Ar. ramidus now confirms that extant African apebased models are no longer appropriate.

Adaptive suites. An alternative to referential modeling is the adaptive suite, an extrapolation from optimization theory (35). Adaptive suites are semiformal, largely inductive algorithms that causally interrelate fundamental characters that may have contributed to an organism's total adaptive pattern. One for the horned lizard (Phyrnosoma platyrhinos) of the southwesten U.S. serves as an excellent example (Fig. 1) $(36,37)$. For this species, the interrelation between a dietary concentration on ants and its impact on body form imply, at first counterintuitively, that elevation of clutch size and intensification of "r" strategy (maximize the number of offspring by minimizing paternal care) are the ultimate consequences of this specialization (35-37).

Such character and behavioral interdependencies can have profound consequences on evolutionary trajectory, as demonstrated by the equally notable differences in clutch size in the common leopard frog (3500 to 6500 eggs) versus those of numerous species of so-called poison dart frogs 
[typically less than 30 eggs; Table 1 (38)]. To enhance survival of their (as yet) nontoxic offspring, the latter engage in relatively intense male parental investment, a shift that has had a profound adaptive impact on their entire life-history strategy.

The effective power of adaptive suites is demonstrable by their explanatory success. A virtually identical character constellation to that of the horned lizard has been discovered in an unrelated Australian ecological vicar, Moloch horridus (37), which is also an ant specialist. Even given such unexpected consilience, however, adaptive suites are obviously speculative, even for living organisms. In addition, for extant species, the processes by which current characters have emerged are also necessarily hidden in the past and, therefore, are no more accessible than for extinct taxa. Nevertheless, adaptive suites can serve as organizational procedures by which to examine evolutionary processes with increasing acumen. Of further benefit is the fact that they often pose novel testable hypotheses that might not have arisen without them.

Many key human specializations are related to our reproductive physiology and anatomy; human reproduction is as extraordinary as our dentition, locomotion, and encephalization (39). Although it remains possible that such uniqueness emerged only during the Pleistocene, this is less likely in light of Ardipithecus, which shows very early evidence of a major social transformation (25). Moreover, it is the modern African apes that are most derived in many characters, whereas those which are specialized in human evolution (SCC elimination, bipedality) are now known to have been present near the origin of our clade. Our massive brains are obviously a Pleistocene development, but they are also probably sequelae to other major shifts now more fully recorded in the earlier fossil record. It is therefore possible, even likely, that many physiologies and soft tissue features that do not fossilize may have also evolved early in hominid evolution. If so, why were these characters exaptive to our advanced cognition and singular demographic success?

Notwithstanding the revelations now provided by Ardipithecus, it should be noted that extensive studies of African apes and other primates have provided likely details of the sociobehavioral context from which hominids most likely first emerged $(1,11)$. These details were presumably present in the last common ancestor we shared with the African apes, and they almost certainly included aspects of great ape demography and social behavior, including male philopatry (males remain in their natal group), female exogamy (females transfer from natal group at sexual maturity), and prolonged inter-birth intervals, all cardinal characters of an intense "k" (maximized parental care of few offspring) reproductive strategy $(32,40)$. Moreover, investigations of the behavior of other living primates now provide a wealth of information that allows many contextual details of earliest human evolution to be reasonably hypothesized.

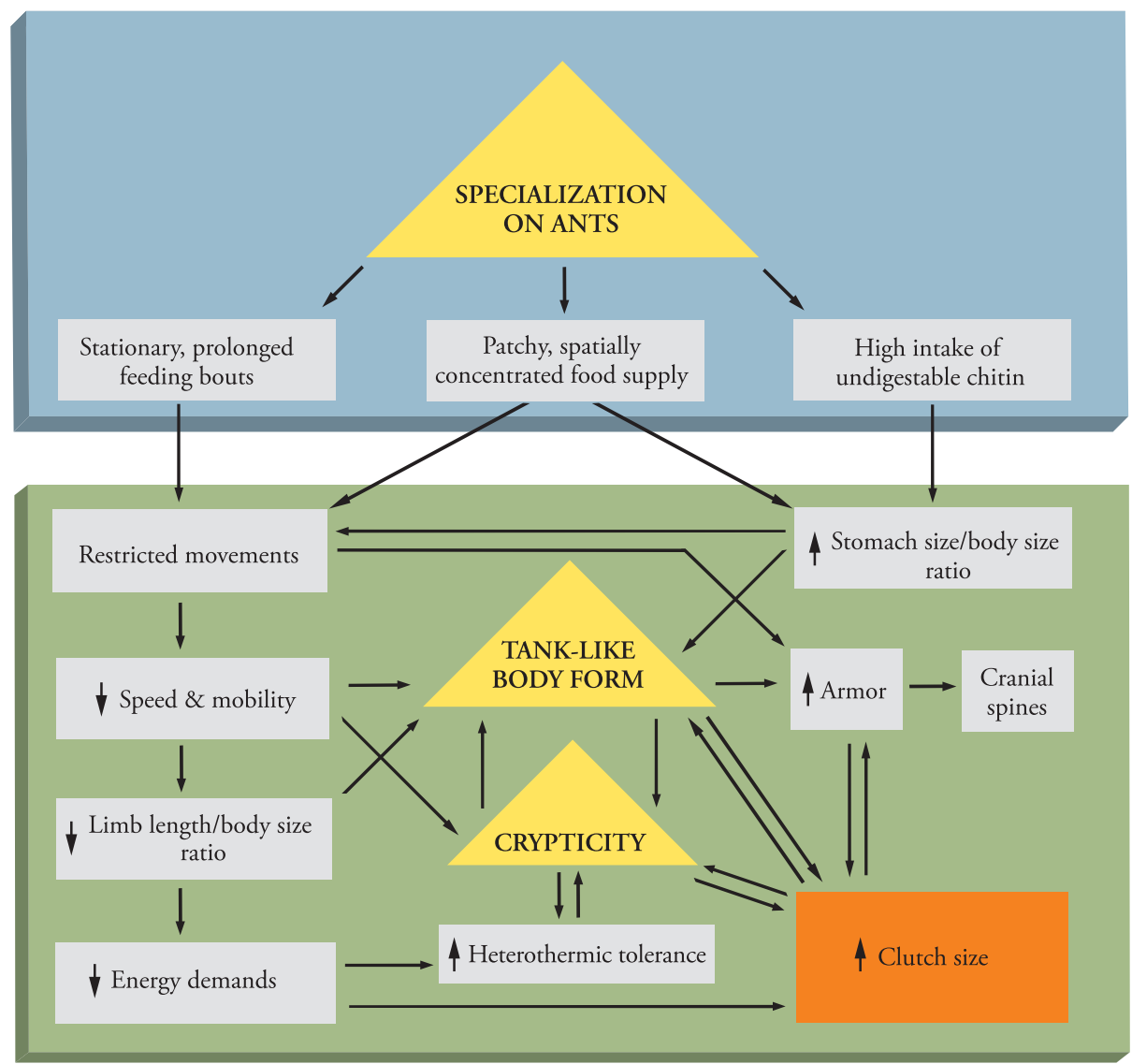

Fig. 1. Adaptive suite of the horned lizard. An adaptive suite summarizes functional interrelations among physiological, locomotor, dietary, and reproductive characters. One is shown here for Phrynosoma platyrhinos. Horned lizards are ant specialists and thus consume copious amounts of indigestible chitin. This requires a large stomach-to-body mass ratio, which in turn generates the lizard's unusual tanklike body form. The latter eliminates flight as an effective predator response, and selection has therefore replaced rapid flight (typical of sympatric lizards) with armor and crypsis (e.g., camouflage). These require motionlessness for long periods, which has generated a tolerance for high variance in body temperature, exceeding that of other sympatric lizards. Motionlessness also relaxes selection against large clutch size (which is very large in $P$. platyrhinos); self-weighting by large clutches in sympatric lizards does not occur because it reduces flight speed [(35), relevant background data available at http://uts.cc.utexas.edu/ varanus/pubs.html].

Table 1. Some differential effects of mating strategy on life-history variables in two amphibians.

Character or behavior Dart frogs*

Clutch size (eggs)

Longevity (years)

Male egg attendance

Male tadpole transport

Female provisioning

$4-30$
$13-15$
yes
yes
yes
Leopard frogst

*Data from (37). †Data from (114).

Sperm competition. Two key factors dominate anthropoid reproductive behavior and are therefore diagnostic of socio-sexual structure: (i) sperm competition and (ii) male-to-male competition for mates. Various anatomical correlates distinguish monogamous or single male primates from other species whose males engage in sperm competition. Among the most obvious is the much higher ratio of testes volume to body mass. Human ratios are generally similar to those of monogamous gibbons and solitary orangutans, but the ratios are three times higher in Pan $(41,42)$ and other sperm-competing species such as Brachyteles (43). Moreover, human testes are most similar to those of gibbons with respect to their higher proportion of intertubular (nonseminiferous) tissue (42). Mammalian sperm competition is generally accompanied by elevated ejaculate quality (44), which is also notably poor in humans. In Homo sapiens, the absolute rate of sperm production is only about $20 \%$ that of much smaller rhesus macaques (45). Another measure, spermatogenesis efficiency (daily sperm production per gram of testes), "varies from about $2.65 \times$ 
$10^{7}$ in rabbits to $<0.06 \times 10^{6}$ in humans" (46). The estimated corresponding value in chimpanzees is greater than that of humans by two orders of magnitude (42).

The muscular coat of mammalian vasa deferentia can reasonably be regarded as a correlate of sperm transport rate during sexual stimulation. It is substantially thicker in chimpanzees than in humans or orangutans (47). The seminal vesicles of some monogamous primates are inconspicuous, whereas those of multimale (i.e., ovulating females usually mate with multiple males) macaques and chimpanzees are large; those in humans are only of moderate size (39). Whatever the social caveats may be, human ejaculatory rates (along with those of the monogamous genera Aotus and Symphalangus) are lower than those of 20 primate species (including Pan and Gorilla) by one order of magnitude $(48,49)$, and human sperm counts decrease at ejaculation frequencies of $>4$ per week (50).

Human sperm midpiece volume, which reflects mitochondrial density and motility, falls in the lowest quartile of 21 primate species examined (51). Especially important is the coagulating reaction between some seminal proteins and prostate vesiculase (52). This coagulum, which blocks penetration of competing sperm by forming a vaginal plug, characterizes primates that robustly spermcompete (e.g., Ateles, Brachyteles, Macaca, Pan). This reaction is absent in humans and common marmosets, whose ejaculates are merely gelatinous (53).

The structures of semenogelins I and II (SEMGI and SEMGII) (primary plug coagulates) illuminate the natural history of vaginal plugging. SEMGI suggests a selective sweep in chimpanzees and conversion to a pseudogene in gorillas; humans exhibit neither (52). Together, these data strongly suggest that the social structure in earlier hominids is unlikely to have been typically multimale. This conclusion is supported by recent analyses of primate immune systems, which compared basal white blood cell counts among primates with respect to the likely number of sexual partners as determined by social system (female mating promiscuity). Results showed that "humans align most closely with the [single male] gorilla ... and secondarily with ... [the] monogamous gibbon" [(54), p. 1170].

Humans have the least complex penis morphology of any primate. Complexity is generally associated with multimale social structure (47), and humans lack keratinous penile surface mechanoreceptors that may promote rapid ejaculation that is common in many primates. Finally, humans are the only catarrhine without an os baculum (39).

Competition for mates. If they did not spermcompete, did early hominid males instead compete for single or near-solitary control of female groups? The cardinal indicator of male-to-male agonism in hominoid primates is the SCC. It is regularly employed during both territory defense and dominance disputes. Hominids are often characterized as having reduced canine dimorphism (55). Such reduction is only a secondary consequence of the primary hominid character, which is elimination of the SCC in its entirety. The SCC is not male-limited; that is, it is always expressed in both sexes of all anthropoids, even in species with reduced dimorphism (e.g., some New World atelines). Although females may express the SCC for advantage in conflicts with other females, they principally express its underlying structure themselves because amplification in their male offspring (presumably by androgens or reduced estrogens) enhances their fitness. Hylobatid canine monomorphism is sometimes erroneously confused with that of hominids, but gibbons evolved amplification of the female canine. Ar. ramidus shows that elimination of the SCC in hominids is unique among all higher primates and occurred long before Australopithecus.

A frequent explanation of canine reduction (and bipedality) is that hand-held weapons replaced the SCC $(56,57)$. But if male-to-male agonism had been fundamental to early hominid fitness, what selective agency would have reduced its signature character? Additional human attributes belie the improbability of the weapons argument. An absence of sperm competition in gorillas and orangutans is accompanied by a dramatically reduced testes size and the elimination of a free scrotum (their testes are more judiciously sequestered in a post-penial bulge) $(42,58)$. In contrast, not only are human scrota more pendulous than even those of chimpanzees (58), but bipedality makes them extraordinarily vulnerable during upright combat (59). It seems illogical to attribute habitual uprightness to weapons that would demand even greater selection for testes sequestration than is present in other primates [which target them with their functional SCCs (60)].

Available evidence now suggests that the loss of the SCC was, as is theoretically most likely, a social adaptation. This evidence, derived from Ardipithecus, includes the following (25): (i) Change in the more socially important upper canine preceded that in the lower, (ii) progressive shape modification made the canine not only smaller but less weaponlike in form, (iii) male canines erupted relatively earlier than in largecanined species with high male-to-male agonism, making this event less likely to have represented a social signal of male maturity, and (iv) all of these changes took place within a dietary context that preceded any of the profound changes seen in later hominid dentitions.

Humans are also unique among primates in lacking vocal sacs, which play a major role in the territoriality of all apes. Though there are no current means by which to judge the evolutionary history of the hominoid vocal apparatus (61), it does have potential developmental interactions with basicranial patterning, including an impact on location of the foramen magnum. The dramatic anterior translation of this foramen during the Plio-Pleistocene is almost certainly a corollary of cerebral reorganization and/or expansion (62). However, early hominid vocal apparatus reduction may have influenced initial differential trajectories of cranial form, currently only just detectable in P. paniscus and Ar. ramidus (29). Both cerebral reorganization and facial patterning are clearly central elements of that trajectory, and early reduction of vocal tract mass is thus a potential modulating factor, particularly because it is a possible social corollary of loss of the SCC.

It has long been argued that Australopithecus was unusually dimorphic in body size, implying a largely single-male group structure, but this hypothesis has been biased by comparisons of temporally and geographically disparate samples (63). Of greater importance are (i) the absence of any useful correlation between body-size dimorphism and social structure in hominoids, because both chimpanzees and gorillas exhibit intense male-to-male agonism but exhibit opposite polarities in skeletal dimorphism (63); and (ii) the fact that male body size in many primates is not associated with competition for mates. Rather, it is equally likely to be an ecological specialization derived from reduced size of females (64) and/or male enlargement by selective agencies unrelated to mate acquisition. In any case, Ar. ramidus now transcends the debates over dimorphism in early Australopithecus because available samples indicate that it was minimally dimorphic, suggesting that this was the primitive hominid condition $(19,25)$ and that dimorphism increased in later hominids (see below).

Reproductive biology of the CLCA. Apes radiated profusely during the Middle Miocene ( $\sim 16$ to $11.5 \mathrm{Ma})$ yet became largely extinct by its terminus $(5.3 \mathrm{Ma})$, which coincided with the radiation of Old World monkeys (65). The nearly total replacement of great apes by cercopithecids is likely to have been closely associated with advanced $\mathrm{K}$ specialization in the former, shared by all surviving hominoids $(66,67)$. However, in dramatic contrast to all other ape descendants, hominids became remarkably ecologically and geographically cosmopolitan. What reproductive strategy permitted such success? Equally as important, what was the likely reproductive strategy of the species that was immediately ancestral to both the hominid and chimpanzee clades?

Advanced K selection must have heavily affected the sociobiology of the earliest hominids. K-driven protraction of life history and increased social adhesion require behaviors that avert inbreeding: either isolation of adults as pairs or female transfer among larger social units (68). The latter proscribes male philopatry (males remain in their natal group) and kin selection (individual fitness is amplified by that of relatives) and greatly reduces female-to-female cooperation and its benefits (e.g., alloparenting), placing at a premium novel mechanisms that can enhance parenting. 


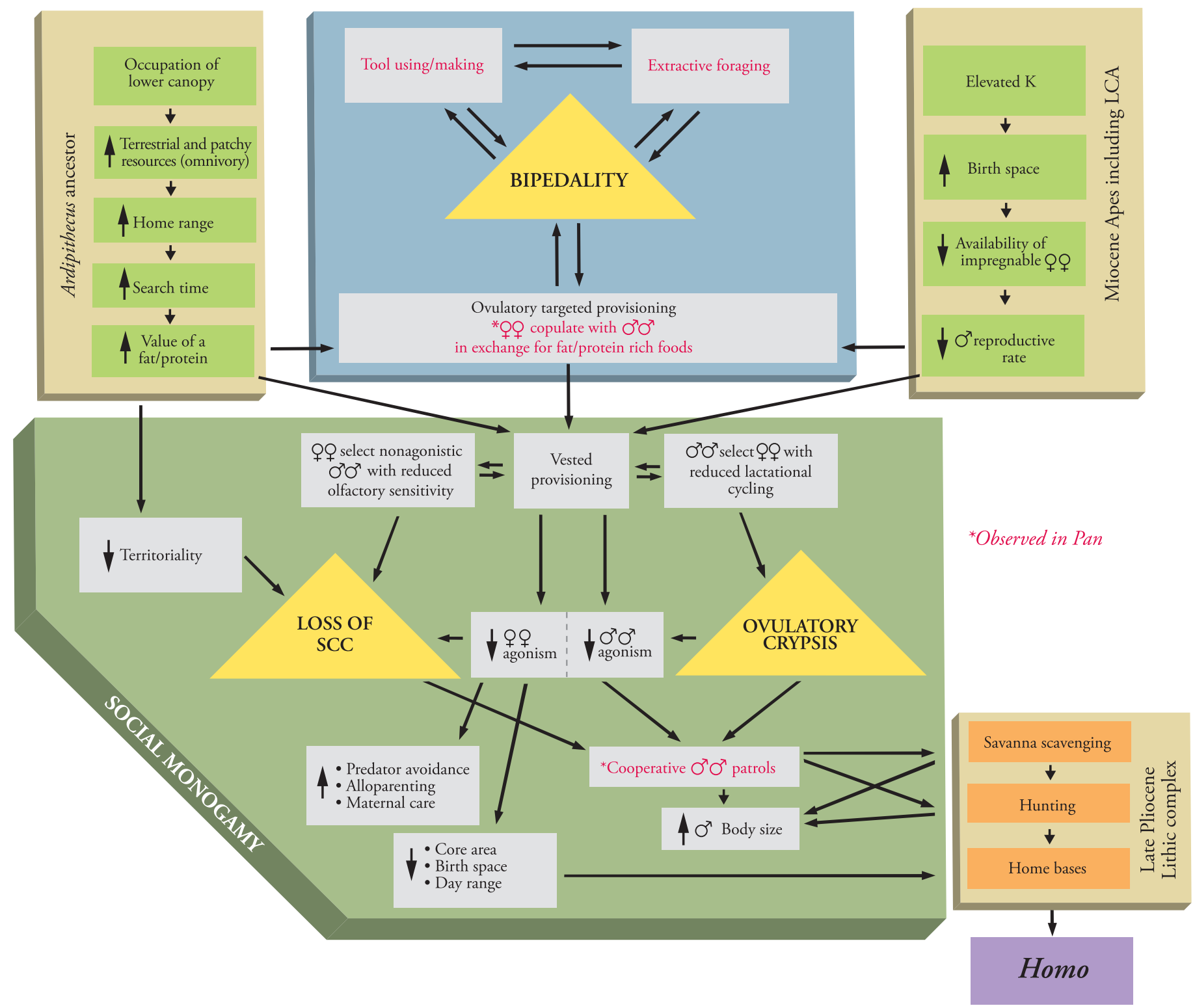

Fig. 2. Emergent adaptive suite in basal hominids. The last common ancestor (LCA) of humans and African apes probably exhibited multimale, multifemale (i.e., females mate with multiple males and vice versa) structure with moderate canine dimorphism and minimal male-to-male agonism, perhaps similar to New World atelines (e.g., Brachyteles), with moderate-to-substantial sperm competition, female transfer (i.e., females leave natal group at maturity), and male philopatry. Here, hominids are hypothesized to have evolved three entirely unique, primary characters (denoted by yellow triangles). Two of these characters, documented in the fossil record, are bipedality and SCC elimination. Modern humans exhibit the third: ovulatory crypsis. Interrelations are hypothesized as follows: (i) transport (object carrying but especially food) leads to habitual bipedality, (ii) female choice of males with limited agonism leads to eclipse of SCC, and (iii) protection against cuckoldry (both sexes) leads to ovulatory crypsis. Two natural sequences generated this adaptive milieu. (Left column) Simple feeding ecology from CLCA to early Ardipithecus and eventually $A u$. afarensis. (Right column) The demographic dilemma $(32,79)$ generated by intensified $\mathrm{K}$ selection. A solution for a hominoid confronting such selective forces is elaboration of sex-for-food exchanges observed in chimpanzees and bonobos. These and other elements shared with Pan acted as possible "social catalysts" [highlighted in red (e.g., copulatory feeding, extractive foraging, male-male patrols)]. Increased male body size and enhanced male-to-male cooperation in $\mathrm{Au}$. afarensis reduced mortality during distanceforaging by multiple-male patrols (whose role was optimal foraging rather than territory defense). This culminated in savanna scavenging, primitive lithics for meat acquisition, marrow extraction, and cooperative hunting in Homo. This profound economic shift selected for advanced adaptations to bipedality, further enhanced social cohesion (reduced same-sex agonism in both sexes), increased energy available for parenting (and alloparenting), promoted survivorship and reduced birth spacing, and elevated the selective ceiling acting against metabolically expensive tissues (e.g., the brain).
The primitive nature of the craniodental and postcranial anatomies of Ar. ramidus suggest that the CLCA, unlike extant African apes, was predominantly arboreal. However, all of its descendants have since developed relatively sophisticated adaptations to terrestrial locomotion (23). What was the CLCA's socio-reproductive structure before these events? Whereas African apes have, in the past, almost invariably been selected as CLCA vicars, Ar. ramidus now allows us to infer that they have undergone far too many pronounced and divergent specializations to occupy such a role.
Possible alternative vicars are extant, K-selected atelines (New World forms such as spider and woolly monkeys), which exhibit many of the CLCA's likely socio-reproductive characters, including male philopatry, minimal-to-moderate canine dimorphism, moderate-to-possibly intense 
sperm competition, regular non-ovulatory based copulation (e.g., Brachyteles), and minimal-tomoderate body-size dimorphism. Minimal maleto-male agonism in some genera probably stems not only from male philopatry, but also from the logistic difficulty of defining and defending exclusively arboreal territories absent special spacing mechanisms and/or dietary adaptations present in related, but less K-adapted genera (e.g., howlers) $(69,70)$.

Exploitation of ground-based resources and terrestrial travel to arboreal ones has encouraged substantial dietary reliance on low herbaceous vegetation in African apes. This has been accompanied by larger home ranges, more intense territoriality, or both. In Gorilla, home range appears to have been secondarily reduced, driven by the body size-dietary axis. These dietary specializations have led to a substantially reduced sex ratio (one to three males per group) and mate guarding in Gorilla, fission/fusion in P. paniscus, and aggressive multimale patrolling, as well as fission/fusion, in P. troglodytes. However, each of these mechanisms substantially discourages male parental investment.

Hominids did not evolve any of the highly derived African ape characters associated with intense male-to-male agonism, reliance on nearground and terrestrial herbivory, or arboreal frugivory (25). Moreover, sperm competition appears to be vestigial in humans [e.g., retained pendulous scrotum, no pseudogenization of SEMGI (52)]. Elaborate periovulatory estrus signaling is therefore most likely a Pan specialization evolved to facilitate female transfer vis-àvis extreme group territoriality and a defense against potential infanticide $(71,72)$, as well as potentially a product of male mate choice in a context of intense sperm competition.

Thus, the hominid and African ape clades evolved wholly divergent social, locomotor, and dietary strategies. Whereas some apes appear to have increased their reliance on terrestrial herbaceous vegetation as early as $10.5 \mathrm{Ma}$ (73), the early hominid dentition remained more generalized $(22,25)$. What unique advantages, then, did bipedality afford only the hominid clade, and how might this unique locomotor pattern be evolutionarily related to elimination of the SCC?

Bipedality and the SCC. Parsimony requires that most, if not all, specialized human attributes emerged within an integrated adaptive constellation, presumably in the same manner as trait complexes in other vertebrates. Figure 2 details one possible adaptive suite as it might have emerged at the base of the hominid clade. Facultative bipedality, a generalized dentition, and enamel isotopic data of $\mathrm{Ar}$. ramidus demonstrate that early hominids continued to exploit both terrestrial and arboreal resources, but in a manner wholly different from those used by extant Pan and Gorilla (25).

Terrestrial resources are more defensible than arboreal ones (74). Terrestriality has obviously elevated resource warding and extra-group male- to-male agonism in Pan and in largely singlemale Gorilla. The elimination of the SCC in very early hominids, however, suggests that resource guarding was not feasible. Territories too large for successful defense have numerous correlates (e.g., patchy resources, elevated search time, enlarged core areas, and increased predator risk) (35). Each of these substantially compromises parenting efforts.

A unique advantage of bipedality is that it permits food transport over long distances, a behavior not generally feasible for an arboreal or quadrupedal hominoid. Bipedality also facilitates the regular use of rudimentary tools, both as carrying devices and as implements for resource exploitation. In a partially ateline-like social structure (but lacking extreme anatomical adaptations to suspension) coupled with a likely early hominid ecological context, females might readily have employed the frequent $P a n$ strategy of exchanging copulation for important food $(11,75,76)$ (e.g., especially valuable meat or fruits high in fat and/or protein), particularly if such items required protracted search time. If obtained by male exploitation of day ranges logistically too large for territorial defense or for effective optimal foraging by females with dependent infants, such dietary items may have become pivotal (77).

The role of tools has, of course, long been a tempting explanation for upright walking (78). However, it is now known that habitual bipedality evolved millions of years before any evidence of stone tools. Despite the potential facility of crude implements of any kind to improve extractive foraging, it remains unlikely that such simple implements would have, alone, been sufficiently critical to reproductive success to have required adaptations (bipedality) that would have simultaneously restricted access to the equally important arboreal resource base. Moreover, both capuchins and chimpanzees effectively transport tools without any reorganization of their postcrania.

On the other hand, the common mammalian and avian strategy of provisioning provides myriad benefits directly associated with reproductive success $(32,79)$. Females and their offspring enjoy reduced predation risk, and males benefit from intensified mothering of their offspring. In such a context at the base of the hominid clade, temporary pair bonds based on sex-for-food exchanges would have further encouraged copulation with provisioning males, rather than males that relied on dominance or aggressive displacement of competitors abetted by large and projecting canines. Research has confirmed the selective advantages of such exchanges in Pan $(11,80)$. Even controlling for rank and age, chimpanzee males that practice meat-for-sex exchanges have elevated fitness levels, and provisioning on a long-term basis improves reproductive success, even after controlling for estrous state (81).

Preference for a dominant male is an obvious female strategy, but it becomes increasingly less favorable when prolonged subadult dependency requires intensified parenting. Under such conditions, survivorship increasingly dominates fecundity. Basal hominid females may have become progressively more solicitous of smaller-canined (and thereby less agonistically equipped) males, particularly if they could encourage such males to habitually target them in preference to other females. Temporary or occasional coupling [including honeymoon pairs (80)] and male choice of particular females for such targeted provisioning would have increased their probabilities of both paternity and subsequent offspring survivorship, which is exceptionally valuable to the reproductive success of both participants.

Any mammal species undergoing advanced $\mathrm{K}$ modifications must eventually approach a limit at which male parental investment becomes virtually mandatory.

Typically, male mammals ... do not form bonds with offspring or mates, and their social relationships are characterized by aggressive rather than affiliative behavior. However, in $<5 \%$ of mammalian species ... ecological demands, such as patchy resource distribution, a low population density of females, or increased predation risk, mean that a promiscuous strategy is not possible. In such species, males are monogamous and contribute to offspring care to safeguard their investment in reproduction.... Although it might seem that the evolution of monogamy in males would require a major reorganization of the brain, recent research has shown that the transition from promiscuity to monogamy might have required relatively trivial mechanistic changes (82), p. 562.

Late Miocene hominoids probably faced a virtual perfect storm of disparate ecological demands. Increased omnivory elevated search time and exposure to predators. Prolonged lactation amenorrhea made ovulating females increasingly rare because birth spacing was progressively prolonged. What solutions to this dilemma could selection offer? Males might cooperate with kin to aggressively expand their territories and gain greater access to additional reproductive females (e.g., Pan), especially if they developed locomotor skills (vertical climbing) that allowed them to rely on high canopy resources and promoted access to ripe fruit. Alternatively, males might aggressively displace all or most others, even if kin-related, to optimize male-to-female ratios (e.g., Gorilla), especially if diet also permitted minimization of day path length so as to prevent female dispersal during feeding.

A third possibility would have been proliferation of sex-for-food exchanges. These would have made provisioning an available solution for both sexes and would have heightened female preference for nonaggressive, provisioning males with which to have repeated copulations. Unlike the circumstances in the first two solutions (Gorilla 
and Pan), in which the SCC would have been under positive selection pressure, the SCC would have been under moderate negative selection in such a clade, because canine retention would have discouraged provisioning in favor of retaining agonistic strategies of mate acquisition.

Reproductive crypsis: the most unique human character. Elimination of the SCC and the ecological context of Ardipithecus at Aramis, Ethiopia, and earlier sites are consistent with the inference that male provisioning via resource transport (and concomitant terrestrial bipedality) antedated 4.4 Ma. Might such behaviors have first evolved nearer the base of the hominid clade? An obvious issue with the hypothesis outlined above is that Pan males prefer females with prominent signs of active ovulation (estrus). If minimal ovulatory signaling in the earliest hominids was primitive (as it is presumably in Gorilla), why did hominid females not prolong and intensify such signs so as to encourage sex-for-food exchanges?

First, the extreme ovulatory-related displays in Pan appear to be derived, because they are associated with comparatively unique molecular signatures of accompanying adaptations (such as proteins necessary for vaginal plugging) absent in other hominoids, as well as appropriately specialized penile morphology. Second, it is unlikely that copulation offered by a female would be rejected by a male - this would be counterproductive given the substantial variability of the primate menstrual cycle and the rarity of hominoid females available for impregnation. Furthermore, habitual provisioning of a target female, even while still lactating for a dependent infant, would still make the repeatedly attendant male most likely to sire any successive offspring upon first reinstatement of ovulatory cycling.

The latter point is critical. One of the most frequently cited objections to male provisioning in early hominids is the problem of cuckoldry during times that males would have been separated from a selected mate while in search of food $(83,84)$. But ovulation in hominoids is an exceptionally rare event, and it probably occurs only after extensive, 3- to 4-year-long periods during which female lactation amenorrhea prevents it. Male provisioning of rarely but obviously fertile females would enhance his fitness by several means: (i) Regular copulation would probabalistically establish an attendant male as the most likely to sire the target female's succeeding offspring, provided that his mate did not "advertise" her ovulation and/or solicit multiple copulations. (ii) Repeated provisioning would accelerate reinstatement of ovulation by replenishing fat stores depleted by lactation. (iii) Accidental or pathological death of her dependent offspring (a not infrequent event) would also reinitialize ovulation, and selection would obviously favor habituation with nonaggressive males not predisposed to infanticide, which is already unlikely because of philopatry.

To prevent cuckoldry, male provisioning within the context of a multimale group there- fore requires selection of females with reproductive crypsis. That is, males could only succeed by provisioning mates with self-crypsis; they would otherwise be unprotected from female copulation with more dominant/aggressive males while ovulating. Broadly (but not entirely) nonovulatory copulation, as in Brachyteles $(69,70)$, would permit prolonged exclusivity in operational pair bonds, especially when provisioning males showed preference for females who were not observed to copulate with other males (85). In this context, it is therefore relevant that human females do not externally advertise ovulation [other hominoids exhibit some degree of ovulatory swelling, even if minimal (86)] and also fail to exhibit its substantial physiological self-perception, despite moderately elevated proceptivity during ovulation $(39,87)$.

The neurophysiology of mate choice. Pair bonding is rare among mammals $(\sim 5 \%)$. A common criticism of an adaptive suite similar to that shown in Fig. 2 is that the transition to such a reproductive strategy would be behaviorally unlikely, even if it did confer the major reproductive benefits detailed above. But the recently discovered relation between brain neurophysiology and mating behavior in mammals may provide a rebuttal. In particular, the expression patterns of the receptors for the neuropeptides oxytocin (OT), arginine vasopressin (AVP), and prolactin (PRL) are now known to substantially influence mating and parenting behaviors $(82,88)$. Monogamous prairie voles exhibit distinct OT and AVP receptor distributions within the mesolimbic dopaminergic reward pathway [i.e., ventral tegmental area (VTA), ventral pallidum, and nucleus accumbens]. This is the central corridor that is activated in human cocaine addiction, and the transient actions of OT within this pathway are critical to establish mothering behavior in nonmonogamous females.

Both OT and AVP are released centrally during sexual stimulation. Their receptors, abundantly expressed in critical brain areas of monogamous prairie voles (but only minimally so in polygynous montane voles), are activated in concert with dopamine release. This promotes associative relations with other neural signaling, especially stimuli emanating from the olfactory bulb, affecting the medial nucleus of the amygdala, and resulting in the formation of a pair bond. Because OT receptor up-regulation in the ventral forebrain occurs before parturition and mediates mother-to-infant bonding, this pathway has probably been co-opted as a means of encouraging monogamy, given the probable homology of mammalian neuroendocrine circuitry in both sexes $(89,90)$.

AVP receptor distributions in monogamous marmosets $(91,92)$ and titi monkeys $(93)$, as well as in polygnous rhesus macaques (94), parallel those observed in monogamous and promiscuous voles, respectively, confirming that this reward pathway functions similarly in primates. Although receptor distributions such as those now available for some monkey species are not yet available for humans, there are marked parallels in other related cerebral phenomena revealed by functional magnetic resonance imaging (see also below). Brain activity patterns in women who looked at photographs of men with whom they were in love "looked remarkably similar to those observed after cocaine or $\mu$-opiod infusions with heavy activation of the VTA and striatal dopamine regions" [(88), p. 1053]. As predicted, similar patterns were evoked by photographs of their children. PRL concentrations, also involved in the reward pathway, are strongly up-regulated in both rodents and callitrichid males exhibiting paternal care, but not in species lacking it; PRL is elevated in human males immediately before the birth of their first child (95).

Equally notable is the impact of steroid hormones on paternal behavior in rodents and primates, including humans. Testosterone concentrations are suppressed in males by parturition in species with extensive paternal care, including numerous rodents, callitrichids, and humans. Such reductions may prevent aggression toward infants. Estradiol and progesterone, critical to normal maternal behavior, have not yet been surveyed in nonhuman primate males but are known to be elevated in human fathers (96).

An early hominid adaptive suite. On the basis of their relatively advanced states in Ardipithecus, two of the three primary characters unique to hominids (bipedality, loss of SCC) probably extend well back into the Miocene, perhaps almost to the time of the CLCA. The emergence of these characters in combination is consistent with a strategy of increasingly targeted provisioning, as outlined in Fig. 2. Males would benefit from enhanced male-to-male cooperation by virtue of their philopatry, because it would improve not only their own provisioning capacity, but also that of their kin. Foraging could be achieved most productively by cooperative male patrols (homologous to but strategically entirely unlike those of Pan). Provisioning would reduce female-to-female competition by lowering reliance on individual "sub-territories" (as in chimpanzees) and/or resource warding (97) and would improve (or maintain) social cohesion. Fission/fusion of social groups would also be reduced, ameliorating likely novel predation risk and enhancing the stability of core areas. Further musculoskeletal adaptation to terrestrial bipedality would be imposed by the need to carry harvested foods, simple tools for extractive foraging, and eventually altricial offspring lacking pedal grasping capacity consequent to the adoption of permanent bipedality without a substantial arboreal component (as in Australopithecus).

The third primary character shown in Fig. 2, female reproductive crypsis, cannot be directly traced in the fossil record. What can we surmise of its evolutionary history? As noted earlier, a central component of reproductive crypsis is the loss of visually prominent mammary gland cycling (i.e., concealed by permanent fat stores that simulate lactating glands) in humans. A common 
explanation for permanently enlarged human mammae is that they serve as a male attractant because they may signal adequate fat stores for reproduction (98). But why would an attractant be required when female proceptivity is the only limiting factor acting on all other primate males (no matter what the underlying social system)? Again, as noted earlier, the elaborate periovulatory sexual swellings of Pan are an integral component of intense sperm competition, which hominids clearly lack. Moreover, whereas the loss of mammary cyclicity would be unlikely to evolve in Pan [copulation with lactating females is rare (99)], crypticism would not be a barrier in a context of copulatory vigilance within pair bonds $(32,79)$. Moreover, elimination of cyclicity would protect a provisioning (and thereby heavily invested) male from cuckoldry, because prominent mammaries would discourage interest by extra-pair males. The absence of cycling would simultaneously protect females from potential abandonment (79).

An element of human reproductive crypsis not discussed earlier is the reduction of a male's capacity to detect ovulation via olfactory signaling. It is again difficult to ascertain why selection would directly favor a precipitous loss in olfactory capacity. Yet the loss of olfactory receptors has occurred in the human lineage at a much faster rate than in other higher primates $(100,101)$ and is fully explicable as a product of female choice acting within the context of a provisioning strategy. If males could detect ovulation in this manner, provisioning would almost certainly have accompanied such detection, just as it does in Pan when ovulation is so acutely advertised. Ovulatory crypsis would therefore be a key element in maintaining targeted provisioning by a particular (pair-bonded) male.

These kinds of unique, reproductively related characters are often broadly ascribed to an intensification of human social behavior during the Pleistocene (by largely undefined selective mechanisms) or have simply been ignored. But why should we simply presume that these various soft tissue structures and physiologies were not present in Australopithecus, or even in Ardipithecus, particularly when the latter shows that the CLCA was not morphologically or behaviorally chimpanzee-like? Relegating these derived characters to Homo almost certainly requires each to be assigned causation in near total isolation. One of the instructive aspects of adaptive suites is the demonstration of what must almost always be a complex network of character interaction, even in reptiles and amphibians. More often than not, such interconnectivity is likely to far exceed relatively simplistic arguments such as somatic budgeting.

Viewing the sweep of hominid evolution in retrospect, it is increasingly unlikely that upright walking, elevation of skeletal dimorphism (in Australopithecus) despite simultaneous elimination of the SCC, loss of vocal sacs, precipitous reduction in olfactory receptors, development of permanently enlarged mammary glands, loss of ovulatory-based female proceptivity, precipitous reduction in male fertility, unique maintenance of a pendulous scrotum despite substantial reduction in testes size, proliferation of epigamics (sexrelated traits used for male selection) in both sexes [implying mate choice in each $(32,79)$ ], and unparalleled demographic success in a terrestrial primate have all been incidental and unrelated. These are far more likely to be multiple elements within a unique reproductive strategy that allowed early hominids to thrive relative to their ape relatives and could have ultimately accommodated rapid development of the unusually energythirsty brain of subadults in emergent Homo.

Yet a large brain is not our most unique characteristic. Chimpanzees have relatively larger brains than cercopithecoids, which have relatively larger brains than lemurs. However, the combination of SCC elimination, habitual bipedality, and reproductive crypsis (each in itself an extreme rarity) is unique among all known mammals. Conversely, simple brain enlargement is readily explicable in myriad ways. If, for example, the acquisition and control of fire was somehow a causative factor, as has recently been suggested (102), what relations does this singular capacity have to the broad array of other entirely unique human characters that are known to have preceded it in the fossil record? Moreover, does the marked expansion of the human brain itself not signal a unique reproductive strategy rather than a simple physical character or capacity? Among the apes, hominids alone were successful before the major cultural advances of the Pleistocene, and Oldowan stone tools persisted unchanged for almost 1 million years. The molecular and cytological records suggest that hominid cerebral evolution extends deep into time, as extrapolated from the likely evolutionary progression in genes such as abnormal spindle-like microcephaly associated gene (ASPM) (103). The reconstructed history of its evolution suggests marked acceleration "along the entire lineage from the last ape ancestor to modern humans ... [implying that] the human phenotype did not arise abruptly ... but [is] instead the consequence of a lengthy and relatively continuous process" [(104), pp. 491-492].

Conclusion. As Au. afarensis was progressively revealed during the 1970 s, its anatomy and antiquity still permitted a possible chimpanzeelike CLCA. Many models of human origins, largely referential, employed this perspective. Previous nonreferential attempts $(32,79)$ argued that only major changes in the social behavior of Au. afarensis and its ancestors could satisfactorily account for its unique combination of postcranial anatomy and unusual demographic success. The tempo and mode of such hypothetical earlier evolutionary events, however, have remained shrouded from our view. This has led to rejection of the hypothesis by many who preferred the comparative comfort and safety of more referential accounts.
Even as its fossil record proliferated, however, Australopithecus continued to provide only an incomplete understanding of hominid origins. Paradoxically, in light of Ardipithecus, we can now see that Australopithecus was too derivedits locomotion too sophisticated, and its invasion of new habitats too advanced - not to almost entirely obscure earlier hominid evolutionary dynamics.

Now, in light of Ar. ramidus, there are no longer any a priori reasons to suppose that acquisition of our unique reproductive anatomy and behavior are unconnected with other human specializations. The evidence is now conclusive: Elimination of the SCC occurred long before the eventual dentognathic hypertrophy of Australopithecus, and long before the likely horizon at which sufficient reliance on tool use would have encouraged abandonment of food and/or safety in the arboreal substrate. It is far more likely that our unique reproductive behavior and anatomy emerged in concert with habituation to bipedality and elimination of the SCC (Fig. 2). It is also now equally clear that Pan's specialized reproductive constellation has been driven by an entirely different locomotor and dietary history.

We currently know very little about the postcranium of hominids older than Ar. ramidus (e.g., Sahelanthropus, Orrorin) $(105,106)$. More fossils will further advance our understanding of the CLCA, and we anxiously await their discovery. Meanwhile, the opportunity of devising adaptive suites for both species of Pan and for Gorilla - grounded in hypotheses generated in light now thrown on the gorilla/chimpanzee/ human last common ancestor and CLCA by $\mathrm{Ar}$. ramidus as to their locomotion, diet, and social behavior - is an intriguing prospect whose alternative outcomes will probably provide additional revelations.

When viewed holistically, as any adaptive suite requires, the early hominid characters that were probably interwoven by selection to eventually generate cognition now seem every bit as biologically ordinary as those that have also affected the evolution of lizards, frogs, voles, monkeys, and chimpanzees. Comparing ourselves to our closest kin, it is somewhat sobering that the hominid path led to cognition, whereas that leading to Pan, our closest living relatives, did not, despite the near-synonymy of our genomes.

As Darwin argued, the ultimate source of any explication of human acumen must be natural selection (78). The adaptive suite proposed here provides at least one evolutionary map by which cognition could have emerged without reliance on any special mammalian trait. The perspective offered by Ardipithecus suggests that our special cognitive abilities derive from a unique earlier interplay of otherwise commonplace elements of locomotion, reproductive biology, neurophysiology, and social behavior. In retrospect, we are as ordinary as corvids (107) and voles (108), although we are much more 
fortunate, if self-cognition is deemed fortunate. We should never have doubted Darwin in his appreciation that the ultimate source of our matchlessness among mammals would prove commonplace when knowledge became sufficiently advanced. Ar. ramidus now enhances that knowledge. Even our species-defining cooperative mutualism can now be seen to extend well beyond the deepest Pliocene.

\section{References and Notes}

1. J. Moore, in Great Ape Societies, W. C. McGrew, L. Marchant, T. Nishida, Eds. (Cambridge Univ. Press, Cambridge, 1996), pp. 275-292.

2. D. Pilbeam, Mol. Phylogenet. Evol. 5, 155 (1996).

3. D. Pilbeam, J. Exp. Zool. 302B, 241 (2004).

4. R. Wrangham, D. Pilbeam, in All Apes Great and Small Volume I: African Apes, B. Galdikas et al., Eds. (Kluwer Academic/Plenum, New York, 2001), pp. 5-17.

5. R. A. Dart, Nature 115, 195 (1925).

6. D. C. Johanson et al., Am. J. Phys. Anthropol. 57, 403 (1982).

7. T. D. White, G. Suwa, Am. J. Phys. Anthropol. 72, 485 (1987)

8. R. A. Dart, Transvaal Mus. Memoir. (Pretoria) 10 , 1 (1957).

9. P. E. Wheeler, J. Hum. Evol. 21, 107 (1991).

10. K. D. Hunt, Int. J. Primatol. 12, 95 (1991).

11. C. Stanford, Upright: The Evolutionary Key to Becoming Human (Houghton Mifflin, Boston, 2003).

12. N. G. Jablonski, G. Chapman, J. Hum. Evol. 24, 259 (1993).

13. J. G. Fleagle et al., Symp. Zool. Soc. London 48, 359 (1981).

14. M. D. Sockol, D. A. Raichlen, H. Pontzer, Proc. Natl. Acad. Sci. U.S.A. 104, 12265 (2007).

15. J. T. Stern Jr., R. L. Susman, Am. J. Phys. Anthropol. 60, 279 (1983).

16. C. O. Lovejoy, Gait Posture 21, 95 (2005).

17. C. O. Lovejoy, Gait Posture 21, 113 (2005).

18. C. O. Lovejoy, Gait Posture 25, 325 (2007).

19. T. D. White et al., Science 326, 64 (2009).

20. C. O. Lovejoy et al., Science 326, 71 (2009).

21. C. O. Lovejoy et al., Science 326, 72 (2009).

22. C. O. Lovejoy et al., Science 326, 73 (2009).

23. C. O. Lovejoy et al., Science 326, 70 (2009).

24. G. Suwa et al., Science 326, 68 (2009).

25. G. Suwa et al., Science 326, 69 (2009).

26. G. WoldeGabriel et al., Science 326, 65 (2009).

27. T. D. White et al., Science 326, 67 (2009).

28. A. Louchart et al., Science 326, 66 (2009).

29. N. S. Clayton, J. M. Dally, N. J. Emery, Philos. Trans. R. Soc. London Ser. B Biol. Sci. 362, 507 (2007).

30. N. J. Emery, A. M. Seed, A. M. von Bayern, N. S. Clayton, Philos. Trans. R. Soc. London Ser. B Biol. Sci. 362, 489 (2007).

31. K. Sayers, C. O. Lovejoy, Curr. Anthropol. 49, 87 (2008) 32. C. O. Lovejoy, Science 211, 341 (1981).

33. K. Milton, in Food and Evolution: Toward a Theory of Human Food Habits, M. R. E. Harris, Ed. (Temple Univ. Press, Philadelphia, PA, 1987), pp. 93-115.

34. R. M. Alexander, in Biomechanics in Evolution, J. M. V. Raynor, R. J. Wootton, Eds. (Cambridge Univ. Press, Cambridge, 1991), pp. 255-266.

35. E. R. Pianka, Evolutionary Ecology (Harper and Row, New York, ed. 4, 1988)

36. E. R. Pianka, W. S. Parker, Copeia 1975, 141 (1975).

37. E. R. Pianka, in Lacertids of the Mediterranean Basin, E. D. Valakos, W. Bohme, V. Perez-Mellado, P. Maragou, Eds. (Hellenic Zoological Society, Univ. of Athens, Athens, Greece, 1993), pp. 121-154.

38. W. Weygoldt, Zool. Syst. Evolutionsforsch. 25, 51 (1987).

39. A. F. Dixson, Primate Sexuality (Oxford Univ. Press, Oxford, 1998).

40. M. P. Ghiglieri, J. Hum. Evol. 16, 319 (1987).

41. A. H. Harcourt, P. H. Harvey, S. G. Larson, R. V. Short, Nature 293, 55 (1981)

42. R. V. Short, in Reproductive Biology of the Great Apes: Comparative and Biomedical Perspectives, C. E. Graham Ed. (Academic Press, New York, 1981), pp. 319-341.

43. K. Milton, J. Behav. Ecol. Sociobio. 17, 53 (1985).
44. Measures include volume, sperm concentration, total sperm per ejaculate, and sperm motility (45).

45. A. P. Moller, J. Hum. Evol. 17, 479 (1988).

46. E. J. Peirce, W. G. Breed, Reproduction 121, 239 (2001).

47. A. F. Dixson, J. Zool. (London) 213, 423 (1987).

48. A. F. Dixson, in The Integrative Neurobiology of Affiliation, C. S. Carter, I. I. Lederhendler, B. Kirkpatrick, Eds. (New York Acadamy of Sciences, New York, 1997), pp. 42-61.

49. A. F. Dixson, Folia Primatol. (Basel) 64, 146 (1995).

50. M. Freund, J. Reprod. Fertil. 4, 143 (1962).

51. M. J. Anderson, A. F. Dixson, Nature 416, 496 (2002).

52. S. B. Kingan, M. Tatar, D. M. Rand, J. Mol. Evol. 57, 159 (2003).

53. A. F. Dixson, M. ]. Anderson, Folia Primatol. (Basel) 73, 63 (2002).

54. C. L. Nunn, J. L. Gittleman, ]. Antonovics, Science 290, 1168 (2000).

55. J. M. Plavcan, J. Hum. Evol. 39, 327 (2000).

56. S. L. Washburn, Sci. Am. 203, 63 (1960).

57. M. H. Wolpoff, Curr. Anthropol. 17, 579 (1976).

58. R. L. Smith, in Sperm Competition and the Evolution of Animal Mating Systems (Academic Press, Orlando, FL, 1984), pp. 601-659.

59. Flaccid human penis length $(13 \mathrm{~cm})$ is unusually great for a hominoid. Length is $4 \mathrm{~cm}$ in Pongo and $3 \mathrm{~cm}$ in Gorilla. Its erect size is greater in the multimale Pan $(8 \mathrm{~cm})$, but this reflects specialized adaptation to penetrate seminal plugs. Short notes that "(e)ven the pubic hair in the male [human] seems designed to draw attention to the genitalia, rather than to conceal them as in the orangutan and gorilla" [(42) p. 338].

60. In three of four fatal attacks by chimpanzee males on other males at Ngogo and Kanyawara, Uganda (109), one or both testes were described as having been "ripped from scrotum." See also www.esquire.com features/chimpanzee-attack-0409.

61. In (110), Alemseged et al. assert that "[t]he bulla-shaped body [of its preserved hyoid] almost certainly reflects the presence of laryngeal air sacs characteristic of African apes" (p. 300). However, there is no correlation between hyoid inflation and the presence or absence of air sacs in primates. For example, Cercopithecus aethiops lacks air sacs, whereas Cercopithecus mitis exhibits them. We have observed that the hyoid bodies of both species are equally inflated (collections in the U.S. National Museum, Washington, DC, and the Royal Museum for Central Africa, Tervuren, Belgium). See also (111).

62. ]. Biegert, in Classification and Human Evolution, S. L. Washburn, Ed. (Aldine, Chicago, 1963), pp. 116-145.

63. P. L. Reno, R. S. Meindl, M. A. McCollum, C. O. Lovejoy, J. Hum. Evol. 49, 279 (2005)

64. A. B. Taylor, Am. J. Primatol. 43, 1 (1997)

65. W. C. Hartwig, The Primate Fossil Record (Cambridge Univ. Press, Cambridge, 2002).

66. D. R. Begun et al., Science 287, 2375a (2000).

67. J. Kelley, T. M. Smith, J. Hum. Evol. 44, 307 (2003)

68. T. H. Clutton-Brock, Nature 337, 70 (1989).

69. C. B. Possamai, R. J. Young, S. L. Mendes, K. B. Strier, Am. J. Primatol. 69, 766 (2007).

70. K. B. Strier, T. E. Ziegler, Am. J. Primatol. 42, 299 (1997).

71. R. W. Wrangham, N. L. Conklin, C. A. Chapman, K. D. Hunt, Philos. Trans. R. Soc. London Ser. B Biol. Sci. 334, 171 (1991).

72. M. E. Rogers et al., Am. J. Primatol. 64, 173 (2004)

73. G. Suwa, R. T. Kono, S. Katoh, B. Asfaw, Y. Beyene, Nature 448, 921 (2007).

74. In (112), Mitani and Rodman devised an index of territoriality that has some predictive success. Substantial increases in day path length and home range size make resource guarding increasingly unlikely.

75. P. Gagneux, C. Boesch, D. S. Woodruff, Anim. Behav. 57, 19 (1999).

76. F. B. M. de Waal, J. Hum. Evol. 18, 433 (1989)

77. M. E. Thompson, R. W. Wrangham, Am. J. Phys. Anthropol. 135, 171 (2008).

78. C. Darwin, The Descent of Man, and Selection in Relation to Sex (John Murray, London, 1871).
79. C. O. Lovejoy, in The Origins of Humans and Humanness, D. T. Rasmussen, Ed. (Bartlett and Jones, Los Angeles, 1993), pp. 1-28.

80. C. E. G. Tutin, P. R. McGinnis, in Reproductive Biology of the Great Apes, C. E. Graham, Ed. (Academic Press, New York, 1981), pp. 239-264.

81. C. M. Gomes, C. Boesch, PloS One 4, e5116 (2009).

82. J. P. Curley, E. B. Keverne, Trends Ecol. Evol. 20, 561 (2005)

83. R. Wrangham, in Tree of Origin: What Primate Behavior Can Tell Us About Human Social Evolution, F. de Waal, Ed. (Harvard, Cambridge, MA, 2001), pp. 119-143.

84. M. Cartmill, F. H. Smith, The Human Lineage (Wiley-Blackwell, Hoboken, N], 2009).

85. A. G. Ophir, B. G. Galef Jr., Anim. Behav. 68, 1221 (2004)

86. "Many ...[have assumed] that our hominid ancestors had sexual swellings that were lost over the course of human evolution, some say to promote pair bonding.... [S]wellings are more likely to have been independently evolved in the line leading to chimps and bonobos. Sexual swellings are scarcely detectable in the other Great Apes or among the last surviving relicts of the genus Homo-modern humans. 'Concealed ovulation' (or more accurately, ovulation without advertising) in our own species is not so much a new trait as an extreme manifestation of an old one." [(113), p. 82]

87. G. Miller, J. M. Tybur, B. D. Jordon, Evol. Hum. Behav. 28, 375 (2007).

88. L. J. Young, Z. Wang, Nat. Neurosci. 7, 1048 (2004).

89. K. E. Wynne-Edwards, Horm. Behav. 40, 139 (2001).

90. K. E. Wynne-Edwards, C. ]. Reburn, Trends Ecol. Evol. 15 464 (2000).

91. Z. Wang et al., Brain Res. 768, 147 (1997).

92. A. Schorscher-Petcu, A. Dupré, E. Tribollet, Neurosci. Lett. 461, 217 (2009).

93. K. L. Bales, W. A. Mason, C. Catana, S. R. Cherry, S. P. Mendoza, Brain Res. 1184, 245 (2007).

94. L. J. Young, D. Toloczko, T. R. Insel, J. Neuroendocrinol. 11, 291 (1999).

95. A. E. Storey, C. J. Walsh, R. L. Quinton, K. E. Wynne-Edwards, Evol. Hum. Behav. 21, 79 (2000).

96. K. E. Wynne-Edwards, C. ]. Reburn, Trends Ecol. Evol. 15 464 (2000).

97. ]. Scott, ]. S. Lockard, Primates 47, 199 (2006).

98. D. Morris, The Naked Ape: A Zoologist's Study of the Human Animal (Random House, New York, 1967).

99. W. B. Lemmon, M. L. Allen, Folia Primatol. (Basel) 30, 80 (1978).

100. Y. Gilad, O. Man, G. Glusman, Genome Res. 15, 224 (2005).

101. D. M. Stoddart, Man 21, 514 (1986).

102. R. Wrangham, Catching Fire: How Cooking Made Us Human (Basic Books, New York, 2009)

103. S. Dorus et al., Cell 119, 1027 (2004).

104. P. D. Evans et al., Hum. Mol. Genet. 13, 489 (2004).

105. Y. Haile-Selassie, G. Suwa, T. D. White, in Ardipithecus kadabba: Late Miocene Evidence from the Middle Awash Ethiopia (Univ. of California Press, Berkeley, CA, 2009) pp. 159-236.

106. Y. Haile-Selassie, G. Suwa, T. D. White, Science 303 1503 (2004)

107. K. Isler, C. P. Van Schaik, Biol. Lett. 5, 125 (2009).

108. H. Walum et al., Proc. Natl. Acad. Sci. U.S.A. 105, 14153 (2008).

109. D. P. Watts, M. Muller, S. J. Amsler, G. Mbabazi, J. C. Mitani, Am. J. Primatol. 68, 161 (2006).

110. Z. Alemseged et al., Nature 443, 296 (2006).

111. G. Hewitt, A. MacLarnon, K. E. Jones, Folia Primatol. (Basel) 73, 70 (2002)

112. J. Mitani, P. Rodman, Behav. Ecol. Sociobiol. 5, 241 (1979).

113. S. B. Hrdy, Ann. N.Y. Acad. Sci. 907, 75 (2000).

114. A. H. Wright, A. A. Wright, Handbook of Frogs and Toads (Comstock, Ithaca, NY, 1949).

115. I thank NSF (this material is based on work supported by grant 9910344) for funding and T. D. White, G. Suwa, M. A. McCollum, P. L. Reno, M. A. Serrat, B. A. Rosenman, R. S. Meindl, M. A. Raghanti, K. Sayers, C. Thompson, T. Gregory, and L. J. Hlusko for extensive discussions and editing of this manuscript over the course of 5 years of revisions.

4 May 2009; accepted 31 August 2009

10.1126/science.1175834 